\title{
Functional neuroimaging using UWB impulse radar: A feasibility study
}

\author{
Timo Lauteslager*, Nicoletta Nicolaou*, Tor Sverre Lande ${ }^{\dagger}$ and Timothy Constandinou* \\ ${ }^{*}$ Department of Electrical and Electronic Engineering, Imperial College London, UK. \\ Email: \{t.lauteslager14, n.nicolaou, t.constandinou\}@imperial.ac.uk \\ †Department of Informatics, University of Oslo, Norway. Email: bassen@ifi.uio.no
}

\begin{abstract}
Microwave imaging is a promising new modality for studying brain function. In the current paper we assess the feasibility of using a single chip implementation of an ultrawideband impulse radar for developing a portable and low-cost functional neuroimaging device. A numerical model is used to predict the level of attenuation that will occur when detecting a volume of blood in the cerebral cortex. A phantom liquid is made, to study the radar's performance at different attenuation levels. Although the radar is currently capable of detecting a point reflector in a phantom liquid with submillimeter accuracy and high temporal resolution, object detection at the desired level of attenuation remains a challenge.
\end{abstract}

\section{INTRODUCTION}

Our ability to study brain function non-invasivively is paramount for diagnosis and treatment of a large number of neurological disorders. Current techniques commonly used for functional neuroimaging (fMRI, PET, SPECT, EEG and MEG), are accompanied by either high costs, poor spatial or temporal resolution or require the injection of radioactive isotopes in the blood stream. These limitations often make the existing technologies less desirable for research applications. New neuroimaging methods that are cheaper, mobile and offer higher spatial and temporal resolution are therefore required.

A newly emerging technique that has lately received a lot of attention in the biomedical field, is active microwave imaging. Electromagnetic (EM) waves in the microwave frequency band are non-ionizing and have tissue-penetrating abilities, making them highly suitable for diagnostic applications. Microwave imaging has been studied in both breast cancer detection [1], [2] and stroke imaging [3]-[5]. A recent attempt to detect bleeding stroke using microwave imaging has shown that EM waves in the $857-1670 \mathrm{MHz}$ band transmit through the head and can be used to detect a volume of blood in the brain [5]. However, to perform functional neuroimaging, a high temporal resolution is necessary to detect the fast changes in cerebral haemodynamics. Regional cerebral blood flow and volume are coupled to neural activation (called 'neurovascular coupling') and are therefore an indicator of brain activity [6]. This feature is exploited by fMRI scans and, because of the dielectric contrast between blood and gray matter [7], could form the basis for microwave functional neuroimaging.

Two approaches have dominated the field of microwave imaging: microwave tomography and ultra-wideband (UWB) radar. Microwave tomography attempts to reconstruct the dielectric profile of the object of interest by solving the inverse scattering problem from the measured scattering parameters. A major disadvantage of the technique is the high complexity of the algorithm that is required for reconstruction, as well as the high scanning time, reducing temporal resolution. UWB radar on the other hand, attempts to detect significant scatterers from the time-of-flight (ToF) of reflected signals. Using beamforming techniques, the source of the reflection can be located with multiple antennas. As this method is computationally efficient and robust, it is suitable to detect small and transient changes over time.

UWB radar imaging is most often performed in the frequency domain. Generally, a vector network analyzer (VNA) is used as a stepped-frequency continuous wave radar. Even though this technique is accurate and provides control over the power at each frequency, it is time consuming. Multiple seconds of scanning time per antenna make it impossible for a multi-static antenna array to study fast physiological changes. Impulse radio UWB (IR-UWB) radar addresses this issue by operating in the time-domain. It emits short (subnanosecond) radio pulses, and can be used for accurate ranging at a high speed. The required high sampling rate leads to power hungry and expensive equipment, but recently a company named Novelda (Kviteseid, Norway) has developed a single CMOS chip IR-UWB radar. A clockless, 'continuous-time binary value' design ensures a sampling rate of $40 \mathrm{GHz}$ [8], while maintaining low power consumption. The emitted EM pulses are higher order derivatives of Gaussian pulses, occupying a bandwidth of $2.5 \mathrm{GHz}$ with an adjustable center frequency ranging from 4 to $9 \mathrm{GHz}$. The energy of the emitted pulses is below the noise floor $(<41.3 \mathrm{~dB} / \mathrm{MHz})$, in accordance with regulations by the FCC. This makes the IR-UWB radar completely safe to use for humans. The high speed and low power consumption would make the Novelda radar particularly suitable for use in a portable, low-cost, non-invasive functional neuroimaging device.

The present work is as a feasibility study of using UWB impulse radar for non-invasive measurement of neural activity. This paper is organized as follows: In section II we will describe a numerical model of the brain to predict the attenuation of EM energy. In section III we present experimental results that demonstrate the capability of the Novelda impulse radar to detect objects in a phantom fluid, and compare the attenuation levels to the predicted attenuation.

\section{NUMERICAL MODEL}

When an EM wave is incident on the boundary between two media with different impedances, a fraction of the energy will be reflected and the remaining fraction will be transmitted. The time of flight (ToF) of the reflected wave can be measured, indicating the distance of the object that caused the reflection. To assess the feasibility of detecting a volume of blood in the brain using radar, a simple 1D attenuation model was built. This numerical model was previously proposed [9] and 
TABLE I. LAYER THICKNESS FOR THE 1D ATTENUATION MODEL

\begin{tabular}{cc|cc}
\hline Medium & Thickness $[\mathbf{m m}]$ & Medium & Thickness [mm] \\
\hline Air & 10 & CSF & 3 \\
Skin & 2 & Gray matter & 4 \\
Fat & 2 & Blood & 1 \\
Bone & 7 & Gray matter & 3 \\
\hline
\end{tabular}

validated [10] for UWB radar, and provides a useful tool to predict the total attenuation due to path loss and the resulting power of reflected EM waves.

\section{A. Model description}

The strength of the reflection of an EM wave impinging on the boundary between two tissues depends on the dielectric contrast between both tissues. For biological tissues, intrinsic impedance $\eta$ can be approximated by:

$$
\eta=\eta_{0} \sqrt{\frac{\mu_{r}}{\epsilon_{r}}}[\Omega]
$$

where $\mu_{r}$ and $\epsilon_{r}$ are the relative permeability and permittivity of the tissue, respectively, and $\eta_{0}$ is the intrinsic impedance of free space $(\approx 377 \Omega)$. The dielectric properties of various biological tissues across frequencies were approximated with the Cole-Cole model, using parameters from [7]. When assuming normal incidence, the expressions for intensity of both the transmitted and reflected wave can be derived from the equations for transmission and reflection coefficient [11]. Transmitted intensity $T$ is expressed as a ratio of transmitted and incident power, and reflected energy $R$ as a ratio of reflected and incident power:

$$
\begin{gathered}
T=\frac{S_{t}}{S_{i}}=\frac{\eta_{1}}{\eta_{2}}\left(\frac{2 \eta_{2}}{\eta_{2}+\eta_{1}}\right)^{2} \\
R=\frac{S_{r}}{S_{i}}=\left(\frac{\eta_{2}-\eta_{1}}{\eta_{2}+\eta_{1}}\right)^{2}
\end{gathered}
$$

where $\eta$ is the intrinsic impedance of either medium 1 or 2 (as obtained from equation 1) and $R+T$ equaling 1 . The average power density in a medium, as a function of the propagation depth $z$ is given by:

$$
S(z)=S_{0} e^{-2 \alpha z}
$$

with attenuation constant $\alpha$ given by:

$$
\alpha=\omega \sqrt{\frac{\epsilon^{\prime}}{2} \sqrt{1+\left(\frac{\sigma}{\omega \epsilon^{\prime}}\right)^{2}}-1}\left[\mathrm{~Np} \mathrm{~m}^{-1}\right]
$$

By combining equations 2, 3 and 4, the total attenuation of a specific trajectory through a number of different media can be calculated. For the application of functional neuroimaging, we are interested in the reflection caused by a volume of blood within the cerebral cortex. The trajectory therefore consists of the following media: Air (the matching medium), skin, fat, bone (estimated as 50\% cortical and 50\% cancellous bone), cerebral spinal fluid (CSF), gray matter (GM) and blood. The head was modeled as multiple layers of planar tissue, with parameters as in table I (acquired from various sources). Total attenuation was determined across a range of frequencies $(0.1$ $10 \mathrm{GHz}$ ). Special interest was taken in the attenuation at $4 \mathrm{GHz}$, as this is currently the lowest possible center frequency of the Novelda radar, and will be the operation mode in the phantom experiment.

\section{B. Model results}

Attenuation was obtained for all direct trajectories and plotted versus depth in Fig 1a. The trajectory of interest,

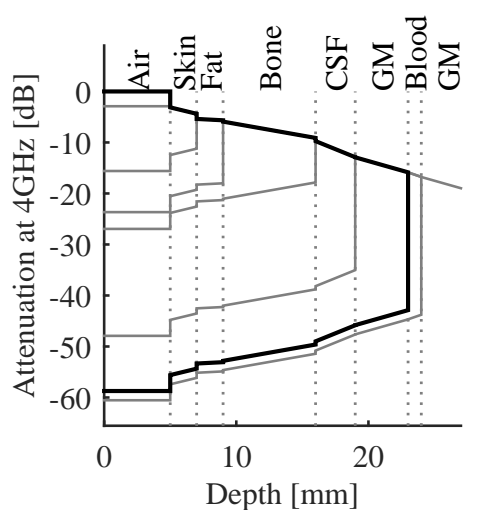

(a)

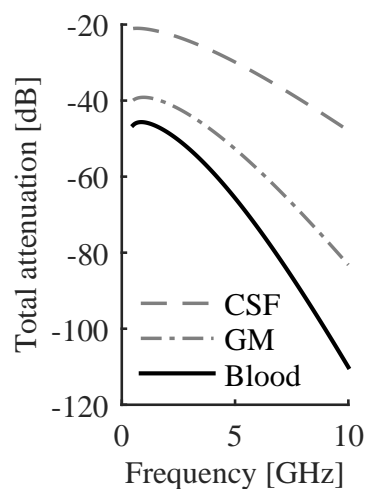

(b)
Fig. 1. Attenuation of EM energy traveling through the head and reflecting off a volume of blood in gray matter (GM). Attenuation is plotted versus depth for all direct trajectories in 1a. Total attenuation is plotted versus frequency in $1 \mathrm{~b}$ for three possible trajectories. The trajectory of interest, caused by a layer of blood, is plotted in black.

which reflects off the blood layer, is plotted in black. A total attenuation of $-58.7 \mathrm{~dB}$ was found at $4 \mathrm{GHz}$. Because attenuation is frequency dependent, the total attenuation is plotted versus frequency in Fig 1b, for the reflections caused by CSF, GM and blood. The attenuation of the trajectory caused by the blood layer is shown in black.

\section{Phantom EXPERIMENT}

To assess the performance of the impulse radar in a controlled but simplified environment, a phantom experiment was performed. This provides a simple way to test the radar's spatial resolution and signal-to-noise ratio (SNR) at certain frame rates.

\section{A. Experimental setup}

A phantom tank was constructed from a standard plastic storage box. A Perspex wall was placed within the box to create a compartment size of approximately $200 \times 310 \times 160 \mathrm{~mm}$, which could be filled with a liquid phantom. The perspex wall was locally thinned down to $1 \mathrm{~mm}$, to reduce the distance of the antenna to the phantom. A solid metal sphere (brass, $25 \mathrm{~mm}$ diameter) was suspended into the phantom liquid and acted as the object. A top mounted caliper was used for accurate object positioning. Two experimental elliptic patch wideband antennas, designed for body-coupling, were attached to the thinned part of the phantom tank wall, at object height, and connected to the Novelda radar. A Mini-Circuits ZX60-V63+ wideband amplifier was connected between the radar's output and transmitting antenna to provide approximately $18 \mathrm{~dB}$ gain. The radar was connected to a pc via USB and controlled through Matlab (The Mathworks, USA).

\section{B. Liquid phantom material}

A liquid phantom (based on recipes from [12]) was created with dielectric properties similar to gray matter at a frequency of $4 \mathrm{GHz}$. To decrease the permittivity of water, 37 parts (by weight) sugar were dissolved in 63 parts water. Additionally, 1 part HEC (Merck Millipore, USA) was added to increase the stability of the solution and 0.1 part Virkon (DuPont, USA) to prevent bacterial and fungal growth. Dielectric properties 


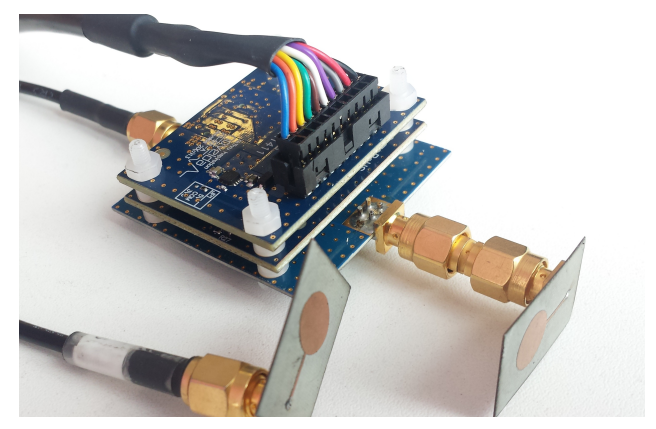

Fig. 2. Novelda UWB radar chip with wideband patch antennas

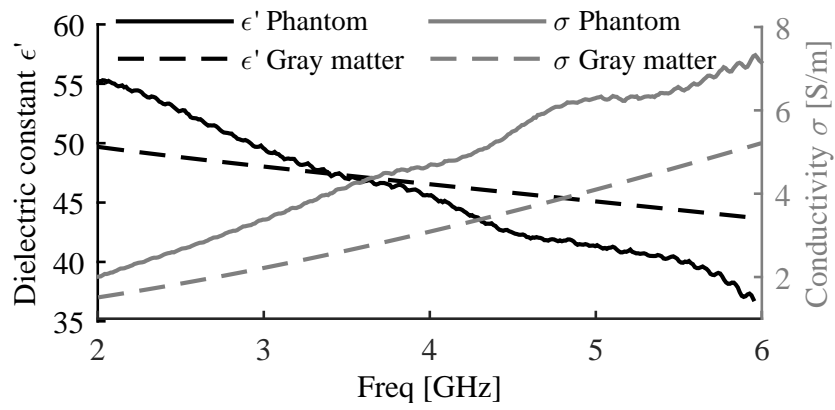

Fig. 3. Dielectric properties $\left(\epsilon^{\prime}\right.$ and $\sigma$ ) of the constructed phantom liquid (solid line) and gray matter tissue according to [7] (dashed line).

of the phantom liquid were measured using a VNA (Agilent E8361A) and dielectric probe (Agilent 85070E) and are plotted in Fig 3.

\section{Protocol}

A number of available radar settings control the trade-off between SNR and the number of frames per second (fps). Data was recorded at a frame rate of $2.6 \mathrm{fps}$. Emitted pulses had a center frequency of $4 \mathrm{GHz}$ and a bandwidth of $2.5 \mathrm{GHz}$. The target object was moved away from the radar in steps of 1 $\mathrm{mm}$, at each step a measurement was made. Data was acquired at a target distance range of $10-25 \mathrm{~mm}$. Target distance was measured from the Perspex wall to the nearest point of the target. In addition to the target measurements, two baseline measurements were performed, without the target suspended in the phantom liquid.

\section{Data analysis}

The Novelda radar consists of 256 parallel samplers with a relative delay of approximately $25 \mathrm{ps,} \mathrm{corresponding} \mathrm{to}$ a sampling frequency of $40 \mathrm{GHz}$. Reflections appear as a delayed and attenuated versions of the emitted signal. The resulting signal is a summation of all reflections, including the direct (not backscattered) path. To illustrate, Fig 4 (top) shows a typical baseline measurement. The direct path can be distinguished as the large Gaussian-shaped waveform, present between samples 0 and 70 . When an object is placed at a distance to the radar, a reflection is discernible, simulated in Fig 4 (bottom) around sample number 200. The waveform is similar to the direct path signal, but lower in power and delayed. When considering the speed of EM wave propagation in air $\left(3 \times 10^{8} \mathrm{~m} / \mathrm{s}\right)$, a sampling rate of $40 \mathrm{GHz}$, and the return path of the signal, the object is estimated to be at a distance of $0.75 \mathrm{~m}$. However, in reality sample number 1 does not necessarily correspond to a distance of $0 \mathrm{~m}$ : the system needs

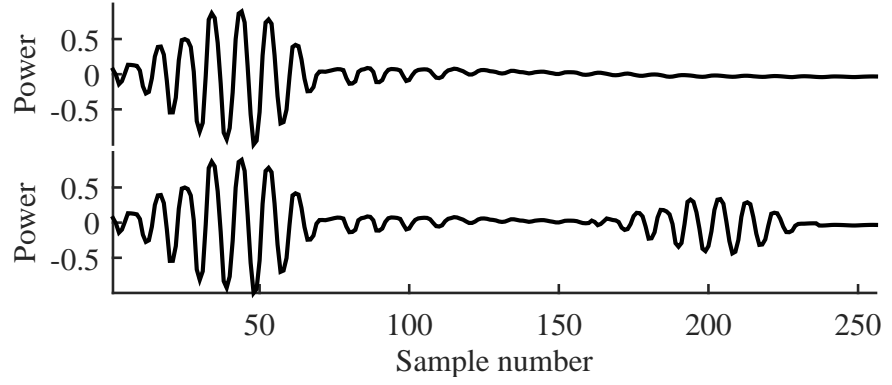

Fig. 4. Illustration of a typical baseline measurement showing the direct (not backscattered) path (top) and a simulated measurement with a reflector placed at a distance of approximately $0.75 \mathrm{~m}$ (bottom). Power was normalized.

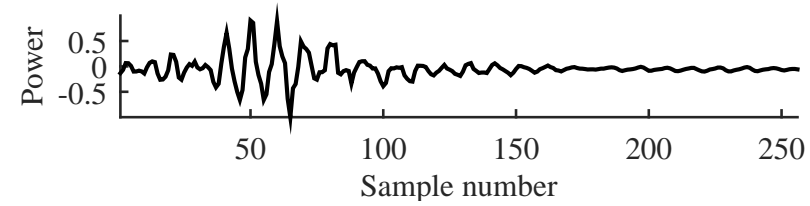

Fig. 5. Example of a recorded signal after baseline subtraction. Identification of reflectors can be done by comparing peak delays between multiple target distances. Power was normalized.

to be calibrated. In addition, the magnitude of the signal of interest will be much smaller than the reflection simulated in Fig 4, and is likely to coincide with the direct path signal due to the short range of the target.

To discern between direct path and target reflection, a baseline measurement was subtracted from all target measurements. The velocity of EM wave propagation in a medium is given by $v=c / \sqrt{\epsilon^{\prime}}$. At $4 \mathrm{GHz}$, the propagation speed in the developed phantom liquid is $44 \times 10^{6} \mathrm{~m} / \mathrm{s}$. This translates to an inter-sample distance of $0.56 \mathrm{~mm}$, which gives our theoretical spatial resolution. When comparing signals recorded at a 1 mm interval, a delay of approximately 2 samples should be expected in the signal peaks. Because the absolute distance of targets in relation to the delay was unknown, these delays were sought for by visual inspection of the data.

\section{E. Experimental results}

After baseline subtraction, recorded signals showed a consistent, low-amplitude waveform (example in Fig 5). Across target distances, peaks were aligned, with the exception of a small number of peaks at sample numbers corresponding to the respective target distances. When comparing target distances 11,12 and $13 \mathrm{~mm}$, a clear delay of peaks (appearing around sample number 80) was detected, as shown in Fig 6. The observed delay of approximately 2 samples was as expected. However, when detecting a target at a distance of $12 \mathrm{~mm}$, total attenuation is estimated at $30.4 \mathrm{~dB}$, according to the numerical model (using the geometry of the experimental setup). At target distances of 21,22 and $23 \mathrm{~mm}$ (estimated total attenuation at $22 \mathrm{~mm}$ : $52.6 \mathrm{~dB}$ ), the delay of peaks is much less obvious (Fig 7 ). The observed peak delay appears around sample number 98, which is a delay of 18 samples relative to signals with a $10 \mathrm{~mm}$ shorter target distance, as expected.

\section{Discussion}

Although the current method of reflection detection (by visual inspection) is very primitive and soon to be replaced by more advanced signal processing techniques, the first 


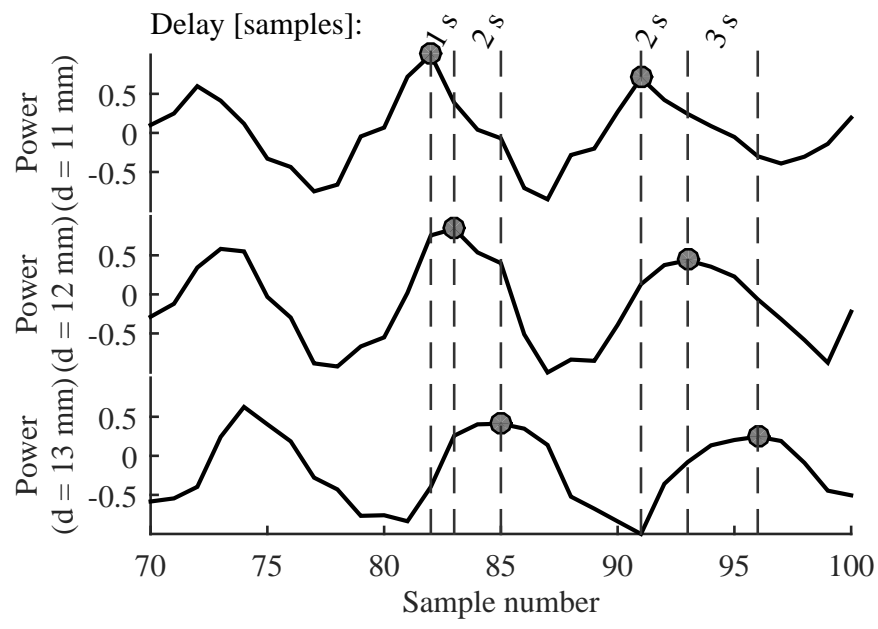

Fig. 6. Subset of samples of measurements with a target at 11 (top), 12 (middle), and $13 \mathrm{~mm}$ (bottom). Baseline measurement was subtracted. Peaks show an average delay of 2 samples, corresponding to the difference in target distance. Power was normalized.

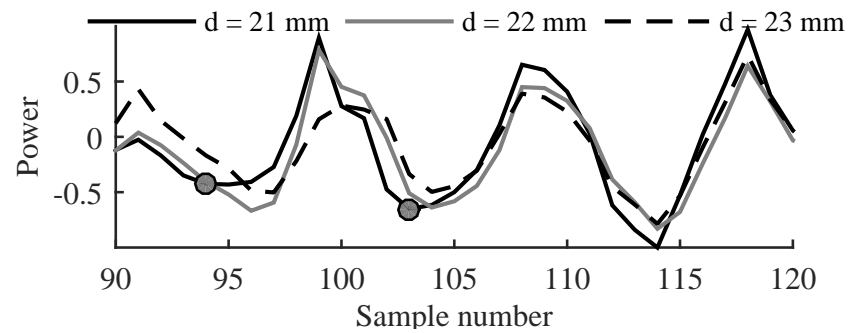

Fig. 7. Subset of samples of measurements with a target at 21 (black solid), 22 (gray solid), and $23 \mathrm{~mm}$ (black dashed). Baseline measurement was subtracted. A delay is discernible but not convincing, due to strong attenuation of EM energy at the measured target distance. Power was normalized.

experimental data is encouraging. The results clearly show the reflections caused by targets at different distances, with a relative delay that is consistent with the difference in target distances. For targets at larger distances $(>20 \mathrm{~mm})$, the reflections become very hard to distinguish. It is promising that the reflections at larger distances do occur at the expected sample number, but clearly more processing gain or a reduction of signal loss is required for reliable target detection. The total attenuation that we estimate to occur in the case of functional neuroimaging is high compared to the levels of attenuation in which we can confidently detect a target in the phantom experiment. We therefore conclude that at this point, object detection at the desired level of attenuation remains a challenge.

The numerical model used for estimating the total attenuation is a very strong simplification of reality. Due to the difficulty of measuring regional cerebral blood volume in humans (MRI contrast agents that are popular in animal studies are not approved for humans), little is known about the magnitude of changes that we can expect in blood volume. A thorough study of the available literature on cerebral haemodynamics and a more advanced 3D FTDT model would provide a stronger assessment of feasibility.

A major contributor to total power loss is the coupling of antenna to medium. The current antennas are designed for efficient radiation into the body while directly contacting the skin. As the human skin has a much higher permittivity than the Perspex wall of our experimental setup, the antenna cou- pling in our experiment was suboptimal. Future investigations will focus to a great extent on antenna coupling, as well as the level of insulation required to minimize near field power losses. Another possible future improvement lies in lowering the center frequency of the emitted pulses. As penetration depth increases with a decrease in frequency, the current center frequency of $4 \mathrm{GHz}$ is rather high. Previous studies on blood detection in the brain using microwave imaging used a frequency band of $857-1670 \mathrm{MHz}$ [5]. In collaboration with Novelda, we aim for a future production of a single chip radar with lower operating frequency. Finally, we intend to add more gain stages, which will lead to a better signal when recording targets at higher depth.

\section{CONCLUSION}

The current experimental setup did not provide enough gain to successfully detect an object at the attenuation levels that we predict for imaging of cortical activity. We have shown however, that the a single chip UWB impulse radar is capable of detecting a small object in a gray matter phantom with high spatial and temporal resolution. Provided that we improve antenna coupling and develop a radar with lower operating frequency, UWB impulse radar is a promising technique for developing a portable and low-cost functional neuroimaging device with high temporal resolution.

\section{ACKNOWLEDGMENT}

The authors would like to thank Novelda for providing hardware, Reza for his help with RF measurements, and Song for lending us his teabox.

\section{REFERENCES}

[1] E. Fear et al., "Microwave breast imaging with a monostatic radar-based system: A study of application to patients," IEEE Trans. Microwave Theory and Techniques, vol. 61, no. 5, pp. 2119-2128, 2013.

[2] E. Porter, A. Santorelli, and M. Popovic, "Time-domain microwave radar applied to breast imaging: Measurement reliability in a clinical setting," Progress In EM. Research, vol. 149, pp. 119-132, 2014.

[3] S. Semenov and D. Corfield, "Microwave tomography for brain imaging: Feasibility assessment for stroke detection," Int J of Antennas and Propagation, vol. 2008, 2008.

[4] R. Scapaticci et al., "A feasibility study on microwave imaging for brain stroke monitoring," Prog In EM Research B, vol. 40, pp. 305-324, 2012.

[5] M. Persson et al., "Microwave-based stroke diagnosis making global prehospital thrombolytic treatment possible," IEEE Trans. Biomedical Engineering, vol. 61, no. 11, pp. 2806-2817, 2014.

[6] K. Kwong et al., "Dynamic magnetic resonance imaging of human brain activity during primary sensory stimulation," Proceedings of the National Academy of Sciences, vol. 89, no. 12, pp. 5675-5679, 1992.

[7] S. Gabriel, R. Lau, and C. Gabriel, "The dielectric properties of biological tissues: Iii. parametric models for the dielectric spectrum of tissues," Physics in med. and biol., vol. 41, no. 11, p. 2271, 1996.

[8] H. Hjortland and T. Lande, "Ctbv integrated impulse radio design for biomedical applications," IEEE BioCAS, vol. 3, no. 2, pp. 79-88, 2009.

[9] E. Staderini, "Uwb radars in medicine," Aerospace and Electronic Systems Magazine, IEEE, vol. 17, no. 1, pp. 13-18, 2002.

[10] G. Varotto and E. Staderini, "A 2d simple attenuation model for em waves in human tissues: Comparison with a fdtd $3 \mathrm{~d}$ simulator for uwb medical radar," in IEEE Int Conf UWB, vol. 3, 2008, pp. 1-4.

[11] J. Kraus, D. Fleisch, and S. Russ, Electromagnetics: with applications. WCB/McGraw-Hill, 1999.

[12] P. Liu et al., "Simulated biological materials at microwave frequencies for the study of electromagnetic hyperthermia," in IEEE EMBS, vol. 1. IEEE, 1992, pp. 272-273. 